\title{
Ketamine: An Update for Its Use in Complex Regional Pain Syndrome and Major Depressive Disorder
}

\author{
William E Bentley*, Michael Sabia, Michael E Goldberg and Irving W Wainer \\ Cooper University Hospital, Camden, New Jersey, United States of America
}

\begin{abstract}
In recent years, there has been significant research in regard to the efficacy of ketamine in regards to treating Complex Regional Pain Syndrome (CRPS) and Major Depressive Disorder (MDD). Many patients have had substantial symptomatic relief; however, not all patients have had positive results. This has prompted current research with evidence to suggest certain biomarkers exist that would infer which patients will/will not respond to ketamine treatment. Current biomarkers of interest include D-Serine (D-Ser) and magnesium, which will be discussed. Whether or not D-Ser and/or magnesium levels should be checked and possibly dictate therapy is an area of future research. It is also possible that downstream metabolites of ketamine are the key to successful therapy.
\end{abstract}

Keywords: Complex regional pain syndrome; Major Depressive Disorder(MDD); Ketamine

Complex Regional Pain Syndrome (CRPS) and Major Depressive Disorder (MDD) are two medical illnesses that may be severely debilitating, have an increased incidence of suicide, and are often resistant to conventional treatments [1]. New research over the past decade regarding the use of ketamine to treat both these diseases has been promising. Ketamine appears to play a role in neuroplasticity and thus may have long-term beneficial effects. It is not entirely clear how this occurs and which patients will benefit from this pharmaceutical therapy. However, data from ongoing pharmacodynamic and pharmacogenetic studies suggest that there are biomarkers that would infer which patients will/will not respond to ketamine treatment.

CRPS is thought to be the result of injury to the autonomic, central, and peripheral nervous systems. It presents as pain affecting one or multiple limbs that does not follow a dermatome distribution and is out of proportion to the inciting injury. The pain may be so severe it can lead to disability. The affected limb(s) are also afflicted with intense changes in skin color, texture, and/or temperature. At times there is neither confirmed nor obvious nerve injury and this is referred to as CRPS-I (previously Reflex Sympathetic Dystrophy Syndrome, or RSD). CRPS-II describes patients who develop CRPS after clearly sustaining nerve damage. However, recent studies have uncovered nerve damage in patients previously classified as CRPS-I, suggesting that there may not be a distinction after all [2].

The pain process of CRPS is multifactorial and conventional treatment is thus multimodal. Common therapies include cognitive behavioral therapy, neuropathic pain medications including pregabalin and gabapentin, physical therapy and occupational therapy, and opioids including methadone. Some forms of CRPS have sympathetically mediated pain and these patients may have significant symptomatic relief from sympathetic nerve blocks. For refractory cases, spinal cord stimulators and intrathecal medications including ziconotide have been implemented with variable success rates. Likewise, ketamine infusions for severe cases of CRPS have had mixed results [3-5].

MDD may also be challenging to treat and ketamine is proving to have therapeutic value for this disease as well. Similar to CRPS, MDD is also a multifactorial disease that has psychological, environmental, and genetic components. The criteria for diagnosis of MDD involves a "distinct change of mood, characterized by sadness or irritability and accompanied by at least several psychophysiological changes, such as disturbances in sleep, appetite, or sexual desire; constipation; loss of the ability to experience pleasure in work or with friends; crying; suicidal thoughts; and slowing of speech and action. These changes must last a minimum of 2 weeks and interfere considerably with work and family relations" [6]. Treatment options include antidepressant medications, cognitive behavioral therapy, and interpersonal therapy. Herbal medications, such as St. John's Wart, have shown similar efficacy as compared to placebo when treating major and minor depression. Refractory MDD therapy includes electroconvulsive therapy [6].

The pathophysiology of CRPS and MDD is not always clear. Likewise, there are many unanswered questions in regards to the effects of ketamine, a drug that has been in clinical practice since 1970. Ketamine is a phencyclidine (PCP) derivative that was developed as an anesthetic agent. Until recent years, ketamine's use has been mostly reserved as an adjunct for anesthesia. At anesthetic doses, it is well known to act as a very potent analgesic, but also produces a cataleptic-like state. Furthermore, recent studies suggest that chronic exposure to ketamine may result in upregulation of $N$-methyl-Daspartate (NMDA) receptors on neurons and this neuroplasticity may lead to a dramatic increase in calcium influx when the receptors are subsequently activated. These high intracellular levels of calcium may lead to neuronal damage or even cell death [7].

A number of recent clinical trials of ketamine have utilized infusions of sub-anesthetic doses in the treatment of CRPS and treatment-resistant MDD, with the added benefit of reducing psychotropic adverse effects as well confining the absolute exposure to ketamine to finite periods of time. In 2005, 40 patients with CRPS received an outpatient ten-day low dose ketamine infusion with a maximum infusion rate of $20 \mathrm{mg} / \mathrm{hr}$. Twenty-five patients received greater than or equal to $70 \%$ reduction in pain at six weeks following treatment [3]. No adverse psychotropic effects were seen during this study. In 2010, 16 patients with CRPS were found to have more profound pain relief after a 5-day inpatient

${ }^{*}$ Corresponding author: Bentley WE, Cooper University Hospital, Camden, New Jersey, United States of America, Hammonton, NJ 08037, Tel: 732-642-6142; E-mail:WEBentley@gmail.com

Received January 26, 2015; Accepted February 26, 2015; Published March 02, 2015

Citation: Bentley WE, Sabia M, Goldberg ME, Wainer IW (2015) Ketamine: An Update for Its Use in Complex Regional Pain Syndrome and Major Depressive Disorder. Clin Exp Pharmacol 5: 169. doi:10.4172/2161-1459.1000169

Copyright: (c 2015 Bentley WE, et al. This is an open-access article distributed under the terms of the Creative Commons Attribution License, which permits unrestricted use, distribution, and reproduction in any medium, provided the original author and source are credited. 
ketamine infusion (with maximum infusion rates $40 \mathrm{mg} / \mathrm{hr}$, which was also well tolerated). A majority of patients experienced "meaningful pain relief" at 6 months following the infusion; however, $37 \%$ of patients denied significant pain reduction [4,5]. Current research also suggests that subanesthetic ketamine may also have a powerful antidepressant effect. Specifically in regards to patients with treatment-resistant MDD, a single $40 \mathrm{~min}$ iv infusion of ketamine $(0.5 \mathrm{mg} / \mathrm{kg})$ produces a rapid (within 4 hours) antidepressant response in a majority of patients that can last greater than seven days $[4,5,8]$.

The therapeutic effect of sub-anesthetic administration of ketamine has been attributed to the direct inhibition of NMDA receptor activity produced by ketamine binding to the intraductal PCP site. This effect has been identified as one of the key pharmacological properties of ketamine. The clinical response was thought to be associated with a chain of events initiated when a patient experiences noxious stimuli, which liberates glutamate from nociceptive afferent nerve endings, which, in turn, stimulates the NMDA receptors causing an influx of intracellular calcium producing a cascade of future neuronal signaling. The repeated, chronic stimulation of the NMDA receptor is involved in the central sensitization seen in chronic pain syndromes. Thus, as an NMDA-antagonist, ketamine decreases the influx of intracellular calcium and downstream signal transmission and thus decreasing proinflammatory cytokines.

However, the clinical effectiveness of ketamine in CRPS and treatment-resistant-MDD patients is varied with about 1 out of 3 failing to achieve an optimal response $[4,5,9]$. Pharmacodynamic studies of ketamine in responding and non-responding patients have indicated that the plasma concentration of ketamine is not the only, or perhaps, key determinant in therapeutic response. This has prompted research into measuring downstream metabolites, distinguishing critical enzymes, and the search for pretreatment biomarkers that will distinguish ketamine responders from non-responders.

Ketamine is extensively metabolized and, therefore, a key factor as to whether or not a patient would receive long term relief from a brief exposure to ketamine may be the systemic exposure of the patient to one or more of these metabolites. One metabolite, nor-ketamine, has been identified as having anesthetic and analgesic properties $[10,11]$ and a second, $(2 \mathrm{~S}, 6 \mathrm{~S})$-hydroxynorketamine has been shown to have CNS activity associated with neurogenesis and antidepressant effects in the rat [12]. Since these metabolites appear to contribute to clinical response, it is logical to assume that the expression of the hepatic metabolizing enzymes, i.e. cytochrome P450 (CYP) enzymes, involved in the production of these compounds would also play a role. While ketamine is metabolized by a number of CYPs, CYP2B6 is a key mediator in the $\mathrm{N}$-demethylation of the parent drug [13]. Since this enzyme is undetectable in up to $15 \%$ of Caucasians and $70 \%$ of Japanese patients [14], pharmacogenetic differences may factor into why certain patients have an unfavorable response to ketamine infusions. This aspect is under investigation in order to determine if pre-treatment genotyping for selective CYP expression can be used to predict ketamine response.

Another approach to the optimization of ketamine treatment is based on the understanding that ketamine exists in two stereoisomeric forms, R-ketamine and S-ketamine. The drug is usually administered as R,S-ketamine, a racemic (50:50) mixture of R-ketamine and S-ketamine. Recently, there has been interest and debate in regards to whether or not the administration of the individual stereoisomers, i.e. $S$-ketamine or $R$-ketamine, may be a more efficacious approach. Initially, $S$-ketamine was presumed to be the drug of choice, as it is ten times more potent at the NMDA receptor $[10,14]$, and this formulation is in clinical use in Europe. However, recent data has suggested that the administration of $R$-ketamine would be preferable in the treatment of MDD as this isomer is free of psycotomimetic side effects and appears to produce more potent and longer-lasting antidepressant effects [15].

The potential clinical use of hydroxynorketamine metabolites in place of the parent drug is of interest based upon their recently observed pharmacological effects $[16,17]$ and the fact that $(2 S, 6 S)$ hydroxnorketamine and (2R,6R)-hydroxynorketamine are primary circulating metabolites after the administration of R,S-ketamine $[14,16]$. It is of interest to note that $(2 S, 6 \mathrm{~S})$-hydroxynorketamine and $(2 \mathrm{R}, 6 \mathrm{R})$-hydroxynorketamine are not potent inhibitors of the NMDA receptor suggesting that there is a different pharmacological mechanism associated with their therapeutic effects. The data from recent studies have indicated that this mechanism involved an indirect inhibition of NMDA receptor activity produced through the reduction in the circulating levels of D-serine, a key co-agonist of the NMDA receptor that specifically activates pre-synaptic NMDA receptors [17]. The therapeutic importance of this effect is suggested by the results from in vitro studies demonstrating that incubation of PC-12 cells with pregabalin and gabapentin reduces the intracellular production of D-serine [18].

D-Serine levels are associated with a number of psychological disease states including neuropathic pain, depression, and chronic pain induced emotional distress, suggesting that this compound might be useful as a pre- and/or post-treatment biomarker of ketamine response. Indeed, a recent study by Goldberg, et al. suggests that basal D-serine plasma concentration may be a biomarker to determine which patients are likely to benefit from ketamine infusions in the treatment of MDD [19]. Using the Montgomery Asberg Depression Rating Scale with greater than $50 \%$ decrease as a positive response, this study noted that decreased baseline levels of $\mathrm{D}$-Ser has a direct correlation with response to treatment $[11,19,20]$.

Magnesium $\left(\mathrm{Mg}^{+2}\right)$ may also play a significant role in the longterm antidepressant and analgesic efficacy of ketamine. There appears to be a direct correlation between low levels of $\mathrm{Mg}^{+2}$ and stress and refractory depression [21]. Nearly all antidepressant medications, including ketamine, increase the levels of circulating $\mathrm{Mg}^{+2}$ in the brain. Intravenous $\mathrm{Mg}^{+2}$ itself has been investigated as a potential therapy. In 2008, a randomized clinical trial showed no difference between $\mathrm{Mg}^{+2}$ versus imipramine in treating diabetic patients suffering from depression [22]. In contrast, in 2001 a double blind randomized controlled trial showed that $\mathrm{Mg}^{+2}$ has no intrinsic analgesic effects in clinically relevant doses [14]. Furthermore, in 2013 a randomized control trial showed no benefit in the treatment of chronic CRPS with $\mathrm{Mg}^{+2}$ versus placebo [23]. However, it is important to note that $\mathrm{D}$-serine is product of the serine racemase mediated racemization of L-serine [24]. Serine racemase is a pyridoxal-5'-phosphate dependent enzyme that requires $\mathrm{Mg}^{+2}$ or calcium $\left(\mathrm{Ca}^{+2}\right)$ for its enzymatic activity. Thus, the importance of circulating $\mathrm{Mg}^{+2}$ concentrations in the therapeutic response to ketamine may be an indirect affect mediated through serine racemase.

In conclusion, the future is bright with the use of ketamine and/ or its metabolites as successful treatment in regards to CRPS and MDD. However, there still is need for much more research. Although $\mathrm{Mg}^{+2}$ has not shown to have any intrinsic analgesic properties [25], it is possible that CRPS patients with low levels may be less likely to respond to ketamine therapy. Randomized controlled trials in regards to baseline $\mathrm{D}$-serine and $\mathrm{Mg}^{+2}$ would help determine if they are in fact potential biomarkers. 
Citation: Bentley WE, Sabia M, Goldberg ME, Wainer IW (2015) Ketamine: An Update for Its Use in Complex Regional Pain Syndrome and Major Depressive Disorder. Clin Exp Pharmacol 5: 169. doi:10.4172/2161-1459.1000169

Disclosure: The authors had no financial support, benefits, nor financial interests in writing this paper. Irving W. Wainer and Michael Goldberg are listed as co-inventors on a patent application for the use of (2R,6R)-hydroxynorketamine and other ketamine metabolites in the treatment of depression and neuropathic pain. Drs. Wainer and Goldberg have assigned their rights in the patent to the U.S. government, but will receive a percentage of any royalties that may be received by the government.

\section{References}

1. Holma KM, Melartin TK, Haukka J, Holma IA, Sokero TP, et al. (2010) Incidence and predictors of suicide attempts in DSM-IV major depressive disorder: a fiveyear prospective study. Am J Psychiatry 167:801-808.

2. Complex Regional Pain Syndrome Fact Sheet. (2013) National Institute of Neurological Disorders and Stroke (NINDS).

3. Goldberg ME, Domsky R, Scaringe D, Hirsh R, Dotson J, et al. (2005) Multiday low dose ketamine infusion for the treatment of complex regional pain syndrome. Pain Physician. 8: 175-179.

4. Goldberg ME, Torjman MC, Schwartzman RJ, Mager DE, Wainer IW (2010) Pharmacodynamic profiles of ketamine (R)- and (S)- with 5-day inpatient infusion for the treatment of complex regional pain syndrome. Pain Physician.13: 379-387.

5. Goldberg ME, Torjman MC, Schwartzman RJ, Mager DE, Wainer IW (2011) Enantioselective pharmacokinetics of (R)- and (S)-ketamine after a 5-day infusion in patients with complex regional pain syndrome. Chirality. 23:138-143.

6. Belmaker RH, Agam G (2008) Major depressive disorder. N Engl J Med. 3: 358:55-68.

7. Mion G, Villevieille $T$ (2013) Ketamine pharmacology: an update (pharmacodynamics and molecular aspects, recent findings). CNS Neurosci Ther 19: 370-380.

8. Luckenbaugh DA, Niciu MJ, lonescu DF, Nolan NM, Richards EM, et al. (2014) Do the dissociative side effects of ketamine mediate its antidepressant effects? J Affect Disord159: 56-61.

9. Zarate CAJ, Singh JB, Carlson PJ, Brutsche NE, Ameli R, et al. (2006) A randomized trial of an $\mathrm{N}$-methyl-D-aspartate antagonist in treatment-resistant major depression. Arch Gen Psychiatry 63: 856-864.

10. Ebert B, Mikkelsen S, Thorkildsen C, Borgbjerg FM (1997) Norketamine, the main metabolite of ketamine, is a non-competitive NMDA receptor antagonist in the rat cortex and spinal cord. Eur J Pharmacol 20:99-104.

11. Leung LY, Baillie TA (1986) Comparative pharmacology in the rat of ketamine and its two principal metabolites, norketamine and (Z)-6-hydroxynorketamine. J Med Chem 29: 2396-2399.
12. Paul RK, Singh NS, Khadeer M, Moaddel R, Sanghvi M, et al. (2014) (R,S)Norketamine and $(2 \mathrm{~S}, 6 \mathrm{~S})$-hydroxynorketamine increase the mammalian target of rapamycin (mTOR) function. Anesthesiology 121: 149-159.

13. Desta Z, Moaddel R, Ogburn ET, Xu C, Ramamoorthy A, et al. (2012) Stereoselective and regiospecific hydroxylation of ketamine and norketamine. Xenobiotica. 42:1076-1087.

14. Sabia M, Hirsh RA, Torjman MC, Wainer IW, Cooper N (2011) Advances in translational neuropathic research: example of enantioselective pharmacokinetic-pharmacodynamic modeling of ketamine-induced pain relief in complex regional pain syndrome. Curr Pain Headache Rep.15: 207-214.

15. Hashimoto K (2014) Blood D-serine levels as a predictive biomarker for the rapid antidepressant effects of the NMDA receptor antagonist ketamine. Psychopharmacology (Berl). 231:4081-4082.

16. Zarate, CA Jr, Brutsche N, Laje G, Luckenbaugh DA, Ramamoorthy A (2012) Relationship of Ketamine's Plasma Metabolites with Response and Diagnosis, and Side Effects in Major Depression. Biol Psychiatry 72: 331-338.

17. Singh NS, Paul RK, Ramamoorthy A, Torjman MC, Moaddel R, et al. (2013) Nicotinic acetylcholine receptor antagonists alter the function and expression of serine racemase in PC-12 and 1321N1 cells. Cell Signal. 25: 2634-2646.

18. Singh NS, Paul RK, Torjman MC, Wainer IW (2013) Gabapentin and (S)pregabalin decrease intracellular D-serine concentrations in $\mathrm{PC}-12$ cells. Neurosci Lett 535: 90-94.

19. Moaddel R, Luckenbaugh DA, Xie Y, Villaseñor A, Brutsche NE, et al. (2014) $D$-serine plasma concentration is a potential biomarker of $(R, S)$-ketamine antidepressant response in subjects with treatment-resistant depression. Psychopharmacology (Berl) 232: 399-409.

20. Wainer IW (2014) Are basal D-serine plasma levels a predictive biomarke for the rapid antidepressant effects of ketamine and ketamine metabolites? Psychopharmacology (Berl) 231: 4083-4084.

21. Murck H (2013) Ketamine, magnesium and major depression--from pharmacology to pathophysiology and back. J Psychiatr Res. 47: 955-965.

22. Eby GA 3rd, Eby KL (2010) Magnesium for treatment-resistant depression: a review and hypothesis. Med Hypotheses. 74: 649-660.

23. Fischer SG, Collins S, Boogaard S, Loer SA, Zuurmond WW, et al. (2013) Intravenous magnesium for chronic complex regional pain syndrome type 1 (CRPS-1). Pain Med. 14:1388-1399.

24. Jirásková-Vanícková J, Ettrich $R$, Vorlová $B$, Hoffman $H$, Lepšík $M$, et al (2011) Inhibition of human serine racemase, an emerging target for medicinal chemistry. Curr Drug Targets 12: 1037-1055.

25. Mikkelsen S, Dirks J, Fabricius P, Petersen KL, Rowbotham MC (2001) Effect of intravenous magnesium on pain and secondary hyperalgesia associated with the heat/capsaicin sensitization model in healthy volunteers. $\mathrm{Br} \mathrm{J}$ Anaesth 86: 871-873. 